\title{
Depletion of a brine layer at the base of ridge-crest hydrothermal systems
}

\author{
Stan Schoofs ${ }^{a}, *$, Ulrich Hansen ${ }^{b}$ \\ a Vening Meinesz Research School of Geodynamics, Earth Science Institute, Utrecht University, Budapestlaan 4, 3584 CD Utrecht, \\ The Netherlands \\ b Institut für Geophysik, Westfälische Wilhelms-Universität Münster, Correnstraße 24, D-48149 Münster, Germany
}

Received 5 October 1999; accepted 6 June 2000

\begin{abstract}
The variable salinity of fluid venting from mid-ocean ridges is indicative of mixing between hydrothermal seawater and fluids that have undergone supercritical phase separation. In order to study the stability of a brine-saturated layer that may form in the lowermost part of the hydrothermal system, we have performed numerical simulations of a system that has returned into the subcritical regime. For typical geological parameters, it is shown that the interface between the brine layer and the overlying fluids is not very stable, but vanishes by one of two dynamical mechanisms: convective breakdown or vertical migration. This contradicts the conventional picture of a steady, layered convective system in which the brine is depleted only by dispersion and diffusion across the interface. The depletion mechanism depends on the fluid-dynamical stability of the brine layer. Convection within the brine layer results either in the convective breakdown (for low excess salinity of the brine, as compared to seawater) or the upward migration of the interface (for higher excess salinities). Consequently, the depletion times are much shorter than for models with pure dispersion/ diffusion across the interface. If the brine layer is static, high-chlorinity liquid is entrained slowly by the convecting overlying fluids, leading to downward migration of the interface. This gradual depletion of the brine layer results in almost constant vent salinities, in agreement with measured salinities of chronic high-chlorinity vents. (c) 2000 Elsevier Science B.V. All rights reserved.
\end{abstract}

Keywords: mid-ocean ridges; geothermal systems; convection; porous materials; brines; numerical models

\section{Introduction}

High-temperature hydrothermal activity is a spectacular feature of the formation of new oceanic crust at spreading centers (see [1] for a re-

* Corresponding author. Present address: GeoDelft, Stieltjesweg 2, 2628 CK Delft, The Netherlands.

Tel.: +31-15-269 3722; Fax: +31-15-261 0821;

E-mail: c.a.schoofs@geodelft.nl view). Seawater penetrates the basaltic crust to a depth of several kilometers, and efficiently transfers heat and chemicals to the seafloor. Direct measurements have indicated that these hydrothermal fluids ventilate through the seafloor at a variety of mass fluxes, temperatures and chemical compositions. A significant amount of heat issues from the crust as hot, so-called 'black-smoker' plumes [2]. Since they are emitted from sources that operate over periods of years, they are also termed 'chronic' plumes. The recorded tempera- 
tures of these plumes fall within the range of $350^{\circ} \mathrm{C}$ to $400^{\circ} \mathrm{C}$, while the chlorinity is generally different from seawater (of which $\mathrm{Cl}^{-}=540$ $\mathrm{mmol} / \mathrm{kg}$ ). On the southern Cleft Segment of the Juan de Fuca Ridge (JDF), the chlorinity of several vents has been stable at values between $\mathrm{Cl}^{-}=951$ and $1087 \mathrm{mmol} / \mathrm{kg}$ for at least a decade [3]. Besides these high-chlorinity vents, many other vents have chlorinities lower than seawater. The chlorinity not only varies between vent sites but may also vary at a single site over time (e.g. [4]). For instance, the chlorinity $\mathrm{Cl}^{-}$of the Monolith black-smoker vent at the northern Cleft Segment of JDF has decreased from 908 to 735 $\mathrm{mmol} / \mathrm{kg}$ over 2 years [3].

An explanation of the variety in the salinities of chronic vents was provided by Bischoff and Rosenbauer [5]. During a magmatic intrusion beneath a mid-ocean ridge, the circulating seawater may become thermodynamically supercritical in the lower parts of the system. When boiling occurs under supercritical conditions, a small amount of highly saline brine forms together with a fresh water phase that has a corresponding reduced salinity. The salinities of both phases depend on the local temperature and pressure conditions [6-8]. The brine salinity may exceed $50 \mathrm{wt} \%$, as found in fluid inclusions in ophiolites [3,9]. It is likely that the buoyant fresh water phase ascends quickly into the overlying (subcritical) fluids, while most of the dense brine stays within the supercritical area underneath $[1,10,11]$. The observed variations in vent chlorinities are well explained by mixing of hydrothermal seawater with either the (condensated) fresh water phase or its complementary brine $[12,13]$.

When a brine layer exists, its evolution plays a significant role in the geological, geochemical and biological evolution of the ridge-crest system. For instance, transport of heat from the magmatic intrusion to the seafloor is influenced by the density interface between the brine layer and overlying fluids $[5,14]$. The brine results also in the spilitization of the lower part of the sheeted dikes and even the underlying gabbros $[9,15]$. The brine chlorinity affects high-temperature chemical reactions between the water and the basaltic rocks and, therefore, the chemistry of vent fluids [16].
Finally, venting of the brine reduces the productivity of most biological communities at the seafloor.

Both the lifetime of the brine-saturated layer and the vent characteristics associated with the brine layer depletion are important factors in these processes. Simple analytical models of the basic physics of brine depletion suggest that the generated brine layer has a lifetime of years to centuries, from the moment that the system has returned into the subcritical thermodynamical regime [10]. However, these methods do not take into account the fluid-dynamical interaction of the brine with the overlying liquids.

In this study, the dynamical processes involved with the depletion of the brine layer are studied numerically once the fluid has returned into the subcritical regime. Previous studies, in which the formation and evolution of convective layers in porous media were investigated under idealized fluid conditions, have shown that a force balance on a fluid parcel just beneath the interface between the two fluid layers determines the stability of layered system $[17,18]$. In the context of the ridge-crest system, this balance states that the stability of the brine layer is mainly determined by (1) the brine salinity established by the thermodynamical conditions, and (2) the hydrological properties of the basaltic rocks that host the boundary. When the restoring force of the chemical interface is larger than the convective forces in the adjacent layers, the interface stays intact and the depletion of the brine occurs by diffusion and dispersion across the interface. Otherwise, the interface can disappear by the migration or breakdown of the interface.

In contrast to those previous studies, the density and viscosity of the hydrothermal fluid at the ridge depends highly and/or nonlinearly on temperature, pressure and salinity. These physical fluid properties invalidate the direct use of the force balance as a quantitative indicator for the stability of the interface. As an alternative, the evolution of the brine layer is studied by a survey of the parameter regime set up by the salinity of the brine and the hydrological properties of the crust. Our simulations start with the emplacement of a brine layer at the base of an already developed 


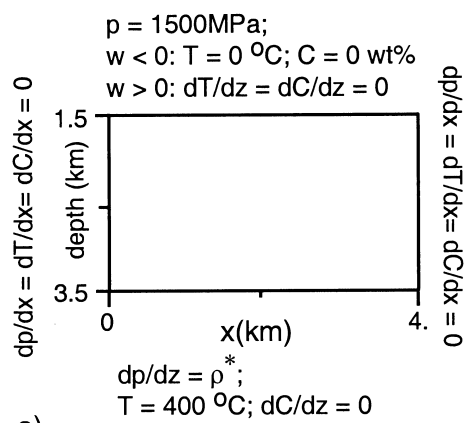

a)

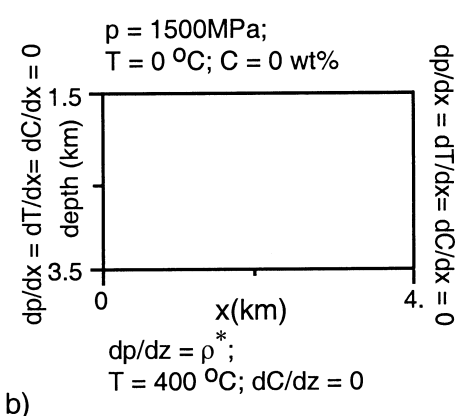

Fig. 1. Geometrical setup and boundary conditions. Two models of a permeable isobaric top boundary condition are considered: (a) $T=0^{\circ} \mathrm{C}$ and $C=3.5 \mathrm{wt} \%$ at points of recharge, and $\partial T / \partial z=\partial C / \partial z=0$ at vent sites. (b) $T=0^{\circ} \mathrm{C}$ and $C=3.5 \mathrm{wt} \%$ everywhere.

layered convective system. Focus is on the dynamical mixing processes between the brine and the overlying fluids. The thermal and chemical vent characteristics associated with these various depletion processes are discussed and compared with observations. Finally, the time scales involved with the depletion are given.

\section{Model setup and formulation}

The ridge-crest hydrothermal system is evidently three-dimensional $[19,20]$. Since thermochemical convection in a low-porosity medium is numerically challenging, however, we feel that a high-resolution study in two dimensions fits our purposes better than a three-dimensional one on a rather coarse grid. Our model resembles a section along the ridge, $2 \mathrm{~km}$ deep and $4 \mathrm{~km}$ wide (Fig. 1). The top is assumed to be $1.5 \mathrm{~km}$ below sealevel and it is isobaric and permeable. Heat destabilizes the liquid, while the solute provides a stabilizing influence.

Two models with different types of top boundary conditions are considered. In the first (hybrid) model, the conditions for temperature $T$ and chemical concentration $C$ depend on whether fluid enters or leaves the domain (see Fig. 1a). The fluid which enters the system has temperature and salinity of seawater. On the other hand, the temperature and salinity of the vent liquid do not alter when crossing the border. The vent plumes which leave the domain through the top are assumed to ascend to the seafloor through perme- able stockworks, without much change of its thermochemical signature [11,21,22]. Overpressures induced by hot and chemical (so viscous) plumes may give rise to sharp pressure variations near the isobaric top boundary. When applying the hybrid boundary condition and a low porosity, this may lead to numerical instabilities. Consequently, at higher permeabilities the use of a second model was inevitable.

In the second model, all fluid at the top has a temperature and salinity of seawater (see Fig. 1b). This constant boundary condition represents a porous medium overlain by an ocean floor. This leaves the disadvantage that also the fluid which flows out of the domain is always cold and has seawater salinity. To overcome this problem, the temperature and salinity at a depth of $200 \mathrm{~m}$ beneath the seafloor are taken as representative values for the vent fluid. The bottom is impermeable and has a fixed temperature of $T=400^{\circ} \mathrm{C}$. The chemical flux through the bottom is equal to zero (i.e. magmatic fluid sources are not considered here). The sides are impermeable and insulators for heat and solute flux.

The starting condition for our simulations is a solution in which convection is well developed in both the overlying fresh and underlying brine layers (for an example, see Fig. 2a). An initially static, layered system would not be appropriate for the problem, since we assume the brine layer has evolved by phase separation before cooling has brought the system back into the subcritical regime. The initial condition was obtained by imposing a barrier to vertical flow $200 \mathrm{~m}$ above the 
base of the domain, separating the two reservoirs, and allowing the model to reach a statistically steady state (the state where average heat transfer has been reached). The resulting temperature, pressure and velocity fields are used as the initial condition, while a uniform brine layer of $200 \mathrm{~m}$ thickness is placed at the bottom and the artificial membrane is removed. A similar procedure was recently followed to develop a layered convective initial condition in simulations of the Earth's mantle [23].

Conservation laws of mass, momentum, energy and chemical concentration of fluid in a rigid porous medium are defined as:

$$
\nabla \cdot \boldsymbol{q}=0
$$

$\boldsymbol{q}=-\frac{\boldsymbol{K}}{\mu}(\nabla p-\rho \boldsymbol{g})$

$\sigma \frac{\partial T}{\partial t}-\kappa \nabla^{2} T+\boldsymbol{q} \cdot \nabla T=0$

$\phi \frac{\partial C}{\partial t}-\nabla \cdot\left(\boldsymbol{D}_{\mathrm{h}} \nabla C\right)+\boldsymbol{q} \cdot \nabla C=0$

with $\boldsymbol{q}$ seepage velocity, $\boldsymbol{K}$ the permeability vector, $\mu$ dynamic fluid viscosity, $p$ pressure, $\rho$ fluid density, $g$ the gravitation vector, and $\phi$ porosity [24]. Although the permeability field in ridge-crest systems is known to be heterogeneous and anisotropic $[25,26]$, the permeability is assumed to be homogeneous and isotropic in this study in order to focus on the fundamental mechanisms involved with the brine depletion. Further, the effective thermal diffusivity of the saturated medium $\kappa$ is taken as a constant. The ratio of the heat capaci- ties between the solid matrix and the fluid $\sigma=\phi+(1-\phi)\left(\rho c_{\mathrm{p}}\right)_{\text {matrix }} /\left(\rho c_{\mathrm{p}}\right)_{\text {fluid }}$ is equal to 0.75 . Here, $c_{\mathrm{p}}$ represents the isobaric heat capacity. Finally, the coefficients of the hydrodynamic dispersion tensor $\boldsymbol{D}_{\mathrm{h}}$ are defined as:

$$
\left(D_{\mathrm{h}}\right)_{x z}=\left(a_{1}-a_{\mathrm{t}}\right) \frac{q_{x} q_{z}}{|\boldsymbol{q}|}+\left(a_{\mathrm{t}}|\boldsymbol{q}|+\frac{\phi D_{\mathrm{w}}}{\tau}\right) \delta_{x z}
$$

where $a_{1}$ and $a_{\mathrm{t}}$ represent the longitudinal and transversal dispersivities, respectively. Further, $x$ and $z$ denote the horizontal and vertical Cartesian coordinates, $D_{\mathrm{w}}$ is the molecular diffusivity of the chemical component within the fluid, $\tau$ the tortuosity of the porous medium and $\delta_{x z}$ the Kronecker delta.

Fluid properties vary with the pressure, temperature and chemical composition of the liquid (for definitions see Table 1). The pressure dependence of viscosity is not included because it is known to be small for subcritical temperatures. Finally, it is assumed that the product $\rho_{\text {fluid }} c_{\text {fluid }}$ is constant, a reasonable assumption since the decrease of density with temperature roughly balances the increase in isobaric heat capacity with temperature [30,31].

A mathematical description of the flow in porous media is defined by Eqs. 1-4 together with the equations of state in Table 1 (for a more extensive description, see [32,33]). A cell-centered grid using a second-order finite volume multigrid method was used to solve the discretized system of equations. The spatial resolution is $256 \times 128$ grid cells, based on extensive testing with different discretizations and time step sizes.

At each time step, the horizontally averaged

Table 1

Fluid properties

\begin{tabular}{ll}
\hline Property/range of variable & Equation \\
\hline Density & \\
$T=0-400^{\circ} \mathrm{C}, p=0.1 \mathrm{MPa}[27]^{\mathrm{a}}$ & $\rho(T)=1028-6.616 \times 10^{-2} T-2.846 \times 10^{-3} T^{2}$ \\
$T=0-400^{\circ} \mathrm{C}, p=1500 \mathrm{MPa}[27]^{\mathrm{a}}$ & $\rho(T)=1086-1.256 \times 10^{-1} T-1.434 \times 10^{-3} T^{2}$ \\
$C=0-100 \mathrm{wt} \%[28]$ & $\rho(C)=1000\left(1.0+7.8 \times 10^{-3} C\right)$ \\
Dynamic viscosity & $\mu(T)=10^{-3}\left(1+1.551 \times 10^{-2}(T-20)\right)^{-1.572}$ \\
$T=0-100^{\circ} \mathrm{C}[29]$ & $\mu(T)=2.414 \times 10^{-5} \times 10^{247.8 /(T+133.15)}$ \\
$T=100-400^{\circ} \mathrm{C}[29]$ & $\mu(C)=1.0 \times 10^{-3}\left(1+1.854 C-1.065 C^{2}+3.00 C^{3}\right)$ \\
$C=0-100 \mathrm{wt} \%[8]$, modified &
\end{tabular}

${ }^{a}$ Appropriate dependence on pressure follows from a weighted interpolation between these two equations. 

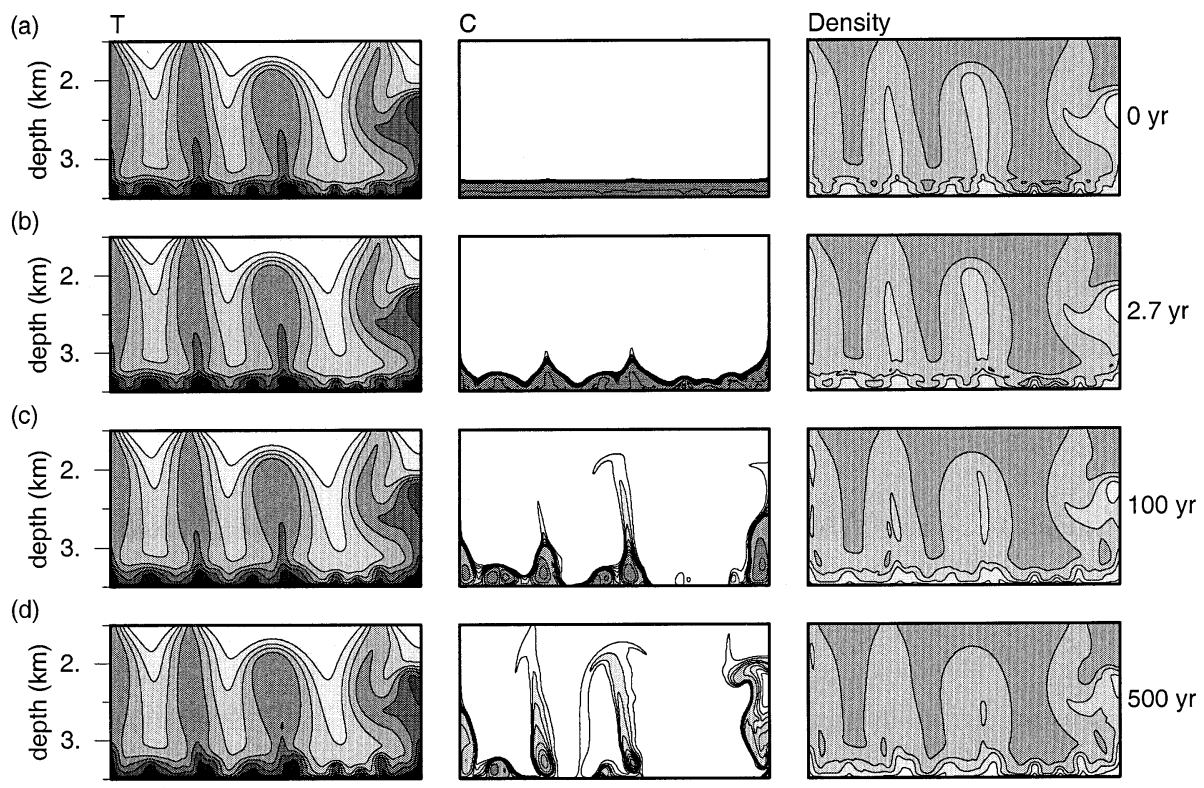

(e)
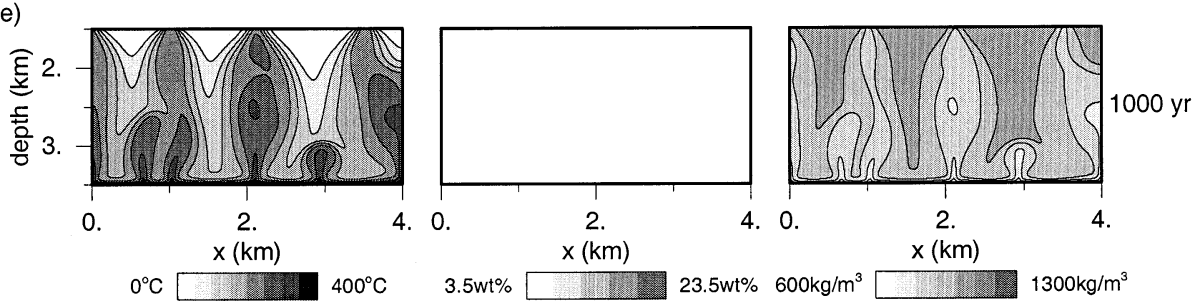

0 .

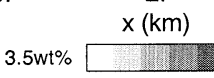

4. 0.

$x(\mathrm{~km})$

Fig. 2. Snapshots of temperature $T$, compositional concentration $C$ and density fields of a simulation with $K=10^{-14} \mathrm{~m}^{2}$, $\Delta C_{\mathrm{e}}=20 \mathrm{wt} \%$ and $\phi=0.01$. At the right, the corresponding times (in years) are given. The contour intervals are $50^{\circ} \mathrm{C}, 2 \mathrm{wt} \%$ and $100 \mathrm{~kg} / \mathrm{m}^{3}$, respectively.

heat and salinity fluxes through the seafloor (hybrid boundary model, Fig. 1a) or at $200 \mathrm{~m}$ below the seafloor (constant model, Fig. 1b) are defined as:

$F_{\mathrm{T}}=-\overline{\lambda \frac{\partial T}{\partial z}+q_{z} T} ; \quad F_{\mathrm{C}}=-\overline{\boldsymbol{D}_{\mathrm{h}} \frac{\partial C}{\partial z}+q_{z} C}$

respectively. Here, $\lambda\left(=2.5 \mathrm{~W} / \mathrm{m}{ }^{\circ} \mathrm{C}\right)$ denotes the thermal conductivity and the overbar implies a horizontal average.

\section{Results}

The base of the system consists of the lower parts of the sheeted dikes and the underlying upper level gabbros. Geologically, this is a very complicated region of which the permeability is poorly known $[21,25]$. The stability and depletion of the brine layer are investigated for the permeabilities $K=10^{-14} \mathrm{~m}^{2}$ and $K=10^{-13} \mathrm{~m}^{2}$, values which may be representative in the region of the brine layer. Within this range, several (if not all) types of dynamical depletion mechanisms are observed. These permeabilities are probably too small to represent the permeability field in the shallower parts of the domain [34,35], but have been taken there for reasons of stability. For $K=10^{-14} \mathrm{~m}^{2}$, the top boundary condition is of the hybrid type (Fig. 1a), while at $K=10^{-13} \mathrm{~m}^{2}$ the top boundary is isothermal and isochemical (Fig. 1b).

In situ measurements [26], analysis of obducted 

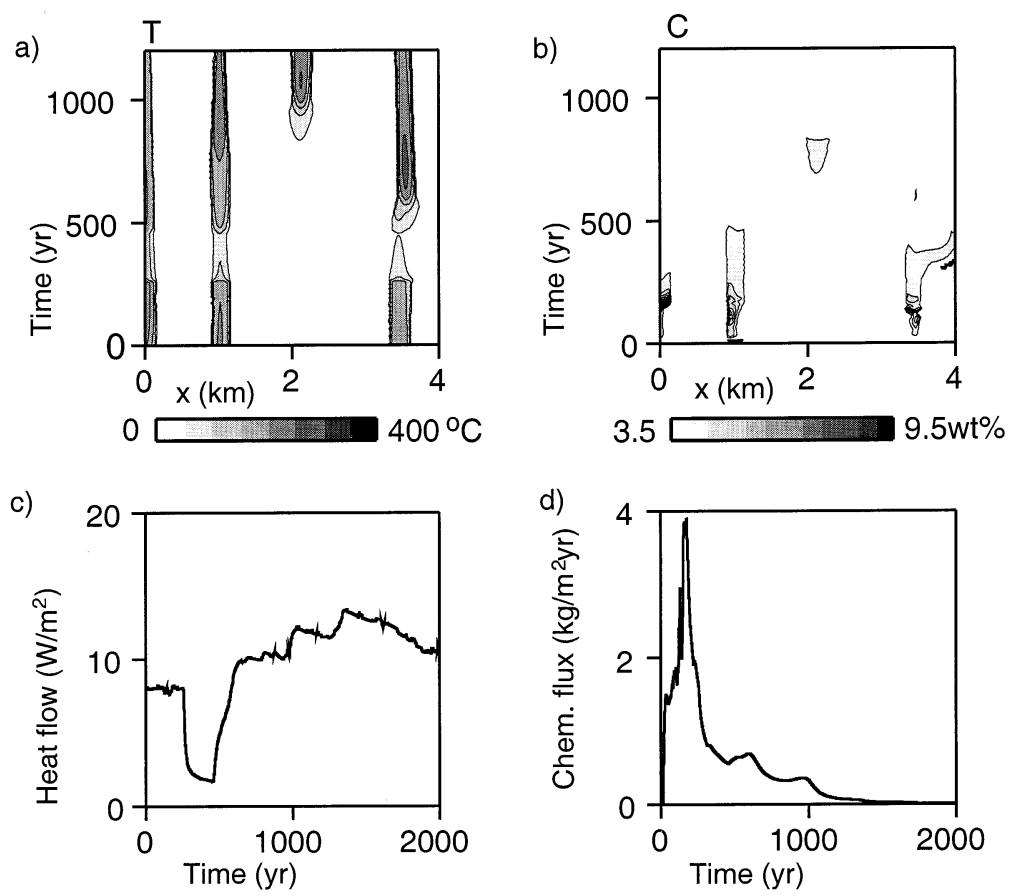

Fig. 3. Temporal evolution of the (a) temperature and (b) compositional concentration at the top boundary, and the horizontally averaged surface (c) heat and (d) solute fluxes, for the simulation depicted in Fig. 2.

ophiolites [25], and theoretical relationships [36] show that effective porosity of the fracture network in the basaltic rocks is of the order of 0.1 to $10 \%$. Since the porosity is a key parameter for the layer-forming process [18,32], we consider both $\phi=0.1$ and 0.01 . The dispersion lengths of the crust are taken as $a_{1}=1 \mathrm{~m}$ and $a_{\mathrm{t}}=0.1 \mathrm{~m}$, values which are reasonable for fractured rocks [37]. For the parameters used, mechanical dispersion dominates over molecular diffusion of the solute within the fluid, while dispersion of heat is negligible. The excess salinity of the brine layer relative to seawater $\Delta C_{\mathrm{e}}$ is varied between 0 and $60 \mathrm{wt} \%$, with intervals of $20 \mathrm{wt} \%$. In total, 16 simulations have been performed.

First, the dynamical processes associated with the depletion of the brine layer are described from the results of three simulations in a medium with a porosity of $\phi=0.01$ (Sections 3.1 and 3.2). In Section 3.3, the sensitivity of the brine-depletion processes to the parameters varied is discussed.

\subsection{Convective breakdown of the interface}

In Fig. 2, the thermal, chemical concentration and density distributions are shown at five stages during the evolution of the layered system, for the parameters $K=10^{-14} \mathrm{~m}^{2}, \phi=0.01$, and an excess salinity of the brine layer of $\Delta C_{\mathrm{e}}=20 \mathrm{wt} \%$. A dark (light) shading indicates a high (low) temperature, salinity or density. Fig. 2a shows the brine layer at the base together with the overlying fluid at the start of the simulation.

The upper part of the domain is characterized by four major plumes, which are separated by regions of cold recharge. Three of these plumes vent through the top boundary, while a cold thermal boundary layer just below the surface keeps the fourth plume from venting. The temperature snapshot shows that several convection cells are present within the brine layer.

The convection currents in the brine layer and in the overlying fluids keep the solute interface sharp but deflect it considerably (Fig. 2b). While 

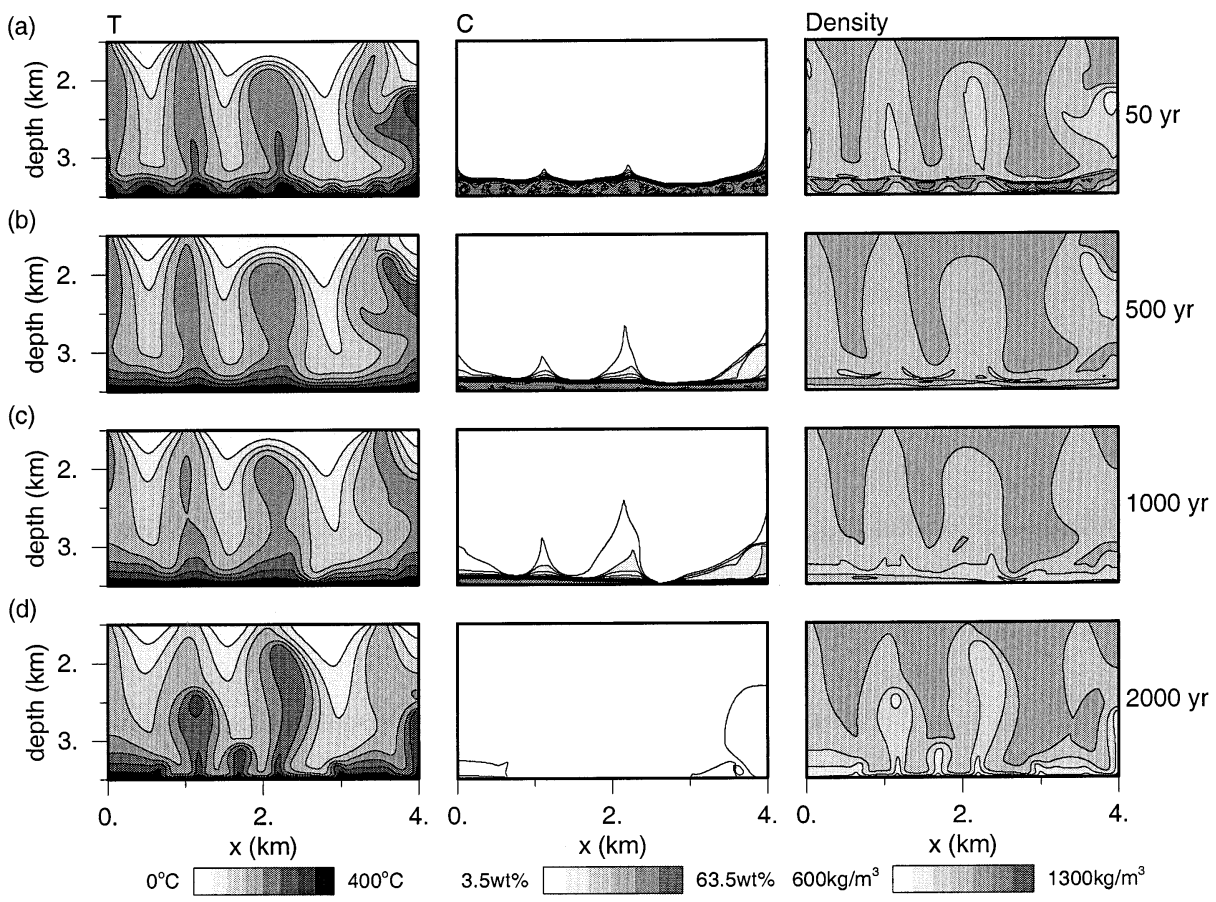

Fig. 4. Temperature, salinity and density snapshots of a simulation with the same parameters as Fig. 2 , except $\Delta C_{\mathrm{e}}=60 \mathrm{wt} \%$. The interface migrates slowly downward, due to the entrainment of brine across the interface. The contour intervals are $50^{\circ} \mathrm{C}$, $5 \mathrm{wt} \%$ and $100 \mathrm{~kg} / \mathrm{m}^{3}$, respectively.

the amplitudes of the interfacial deflections increase quickly, only small amounts of salinity cross the interface by dispersion. At places where the deflecting interface touches the bottom, the brine layer breaks down (Fig. 2c). The separate pieces of the brine layer are entrained into the hot plumes in the seawater layer and, subsequently, they are vented through the seafloor.

The brine which is entrained by the plume that does not reach the surface recycles several times with the circulating seawater. During this period, the brine is mixed with the plume liquid and only a small amount of the brine is lost dispersively through the top (Fig. 2d). Eventually, the plume breaks through the cold upper boundary layer and the remainder of the salinity is quickly flushed out of the domain (see Fig. 2e).

The accompanying top boundary temperatures and salinities are plotted against time in Fig. 3, together with the temporal evolution of the horizontally averaged surface heat and excessive salinity fluxes. The chemical surface data (Fig. 3b and d) show that the major part of the brine vents through the top within $300 \mathrm{yr}$ of the start of the simulation. Furthermore, both the temperature of the vent fluid and the surface heat flux decrease temporarily after this period due to the presence of the interface (Fig. 3a and c).

Although the maximum vent salinities are 9.5 $\mathrm{wt} \%$, which is at salinities three times that of seawater, most of the brine is vented as fluid with salinities no more than two times seawater (see Fig. 3b). After venting of the brine, the position and vigor of the four plumes remain almost constant up to the end of simulation.

\subsection{Migration of the interface}

\subsubsection{Downward migration}

Fig. 4 depicts the $T, C$ and density fields at four stages during the evolution of the system with similar parameters as before, except the excess salinity of the brine layer is increased to $\Delta C_{\mathrm{e}}=60 \mathrm{wt} \%$. The high brine salinity leads to 

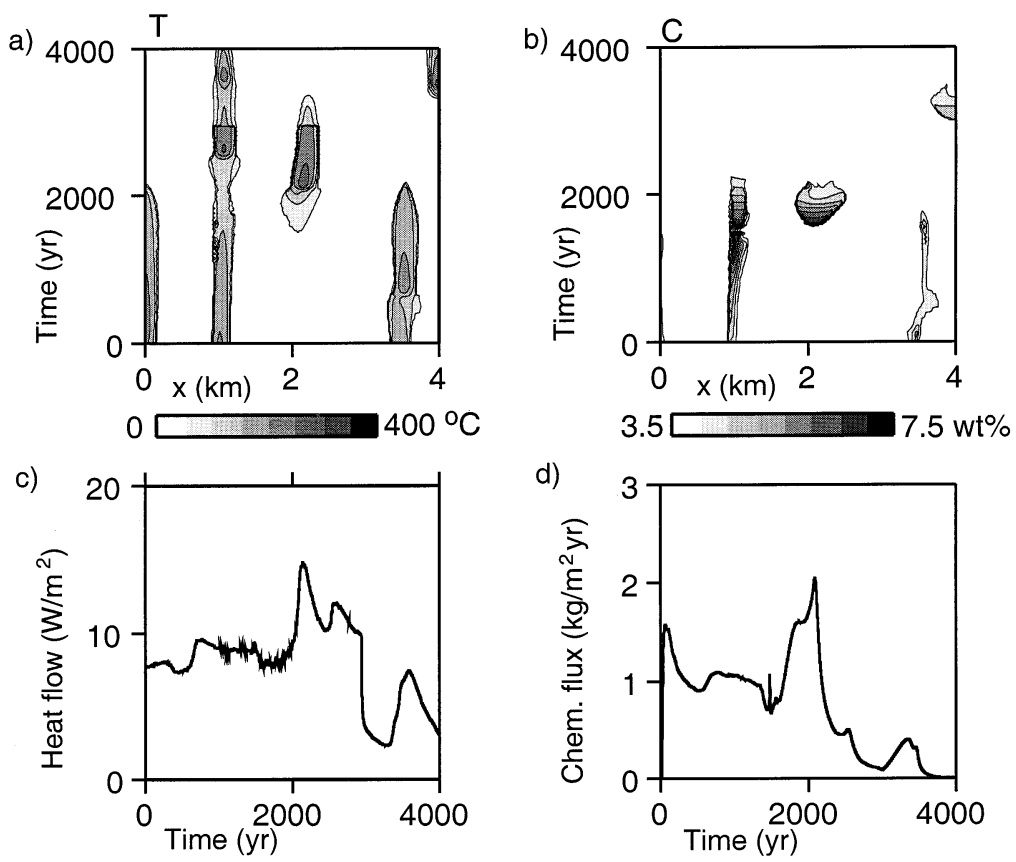

Fig. 5. Temporal evolution of the (a) temperature and (b) chemical concentration at the top boundary, and the horizontally averaged surface (c) heat and (d) solute fluxes, for the simulation depicted in Fig. 4.

density jump of $340 \mathrm{~kg} / \mathrm{m}^{3}$ across the interface. Any deflection of the interface experiences a strong restoring force, and so the interface remains planar (see Fig. 4a). Furthermore, the brine viscosity is 12 times higher than that of seawater under similar $p, T$ conditions (Table 1). As a result, the convective vigor in the brine layer is strongly reduced as compared to the initial situation. It is essentially a thick thermal boundary layer, across which heat is transported diffusively. The near static brine layer is entrained slowly by the overlying seawater and the interface migrates downward (compare Fig. 4a and b).

Although solute is transported continuously across the interface, the salinity difference (and thus the density jump) remains nearly constant because (1) the salinity of the brine layer is distributed homogeneously, and (2) the entrained salinity is transported away from the interface immediately by the convection currents in the upper layer. Consequently, the interface remains flat up to the point that nearly all of the brine has been entrained by the convection currents in the overlying layer and the bottom thermal boundary layer destabilizes (Fig. 4d). At this time, several new plumes develop from the base of the domain.

In Fig. 5, the temperature, salinity and fluxes sampled at the seafloor are plotted. The variation of the surface heat flow is related directly to the number of venting plumes (Fig. 5a and c). Between 0 and 2000 yr Fig. 5b shows stable venting with an almost constant salinity of two times seawater. During this interval, the vent temperatures decrease slightly (see Fig. 5a). The maximum vent salinity is $7.3 \mathrm{wt} \%$. Despite the larger initial brine layer salinity, this value is lower than in the previous simulation.

\subsubsection{Upward migration}

In order to investigate the sensitivity of the stability and depletion of the brine layer to the permeability field, the permeability of the medium is increased to $K=10^{-13} \mathrm{~m}^{2}$. For reasons of numerical stability the second model for the surface boundary (constant $T$ and $C$ ) is employed. In Fig. 6 , the $T, C$ and density fields are shown at five stages during the evolution, in which all parameters except permeability are similar to the simula- 

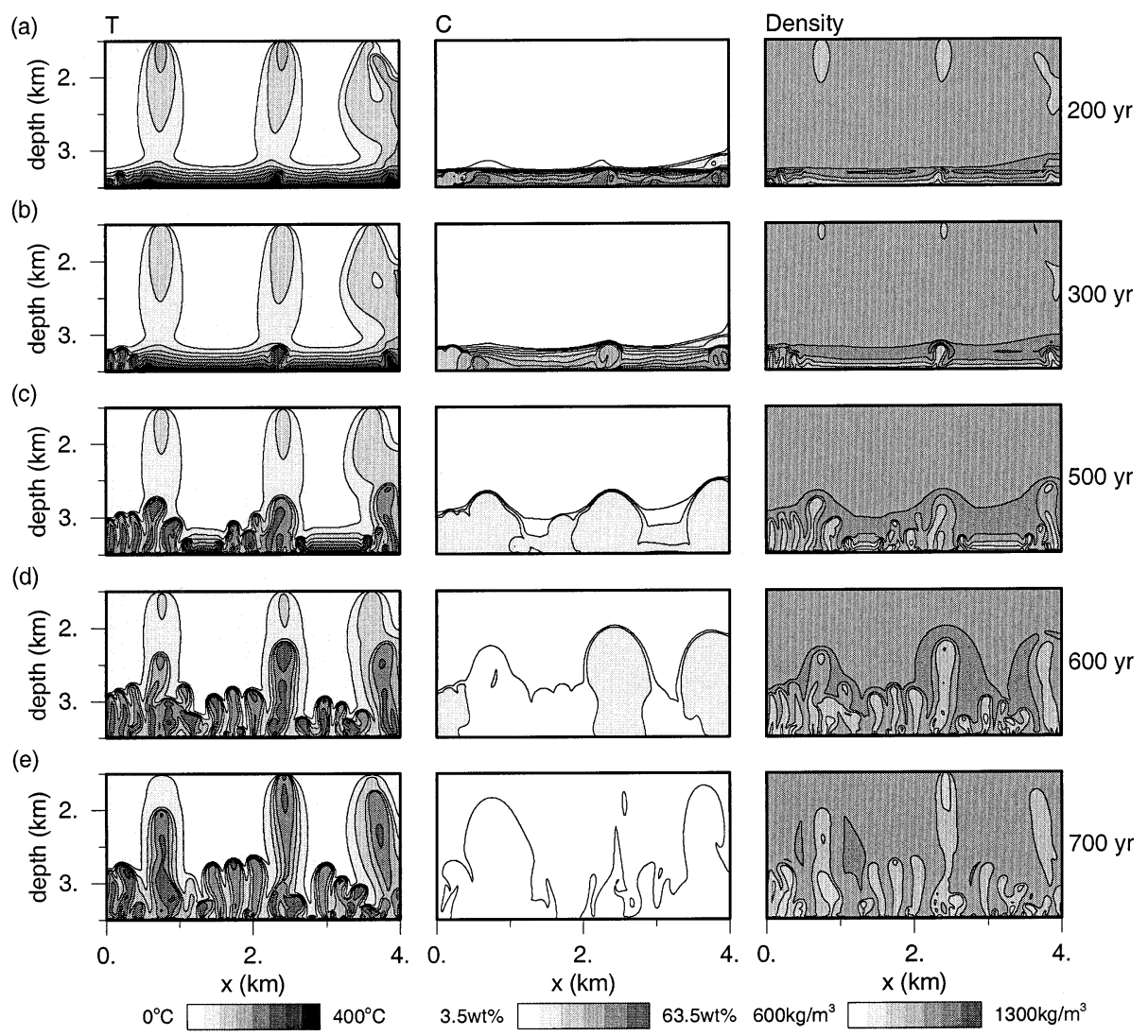

Fig. 6. Snapshot of $T, C$ and density at higher permeability $K=10^{-13} \mathrm{~m}^{2}$, showing the upward migration of the interface due to destabilization of the brine layer. The contour intervals are $50^{\circ} \mathrm{C}, 5 \mathrm{wt} \%$ and $100 \mathrm{~kg} / \mathrm{m}^{3}$, respectively.

tion of Fig. 4. As before, the high viscosity of the brine results in brine layer that is initially almost static (Fig. 6a).

In contrast to the previous case, this (nearly) diffusive brine layer does not remain isolated but breaks down at several locations (Fig. 6a and $b$ ). The convective forces of the new instabilities quickly grow larger than the stabilizing density force of the interface. Due to the nonlinear fluid properties and the migration of the interface, it is not straightforward to determine in which of the two layers convection is most vigorous. Despite a smaller layer thickness, the convective forces in the brine layer appear to be larger than those in the overlying fluids. As a consequence, the interface between the brine and overlying fluids starts to migrate upwards.

Vertical growth of the brine layer by advective entrainment of fresh water from above the interface obviously results in a decreased average chemical content within the layer (Fig. 6b-d). Advective mixing of the brine with the fresh water within the destabilized areas quickly redistributes the chemical content of the brine layer, keeping the layer reasonably well mixed. When the three largest plumes reach the top of the domain, the mixed diluted liquid vents at three sites (Fig. 6e).

In Fig. 7, the temperature and salinity $200 \mathrm{~m}$ below the seafloor are plotted against time (as to represent the temporal evolution of the surface $T$ and $C$ ), together with the horizontally averaged heat and solute fluxes. Fig. 7a shows that the vent temperatures decrease gradually, until the moment that the upward migrating interface reaches the surface. From that moment, all brine flushes out of the domain within a period of 

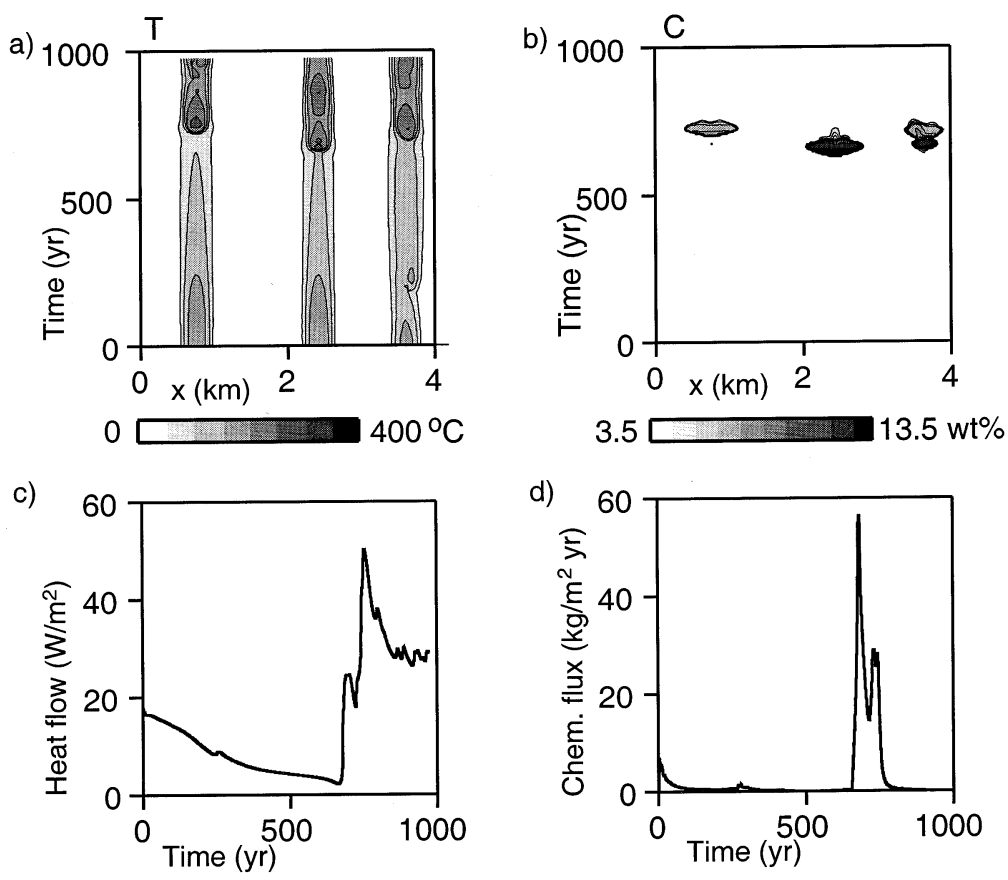

Fig. 7. Temporal evolution of the (a) temperature and (b) chemical concentration at the top boundary, and the horizontally averaged surface (c) heat and (d) solute fluxes, for the simulation depicted in Fig. 6.

around $100 \mathrm{yr}$ (see Fig. $7 \mathrm{~b}$ and d). During this period, the temperatures are high and vent salinities up to $C=13 \mathrm{wt} \%$ are observed.

\subsection{Sensitivity study and depletion times}

In every experiment, the brine layer was depleted by one of the two dynamical mechanisms. Convective breakdown of the interface was observed in the simulations with an excess salinity of $C_{\mathrm{e}} \leq 20 \mathrm{wt} \%$, while interface migration occurred at higher salinities. The direction of the interface migration depends on the convective forces within the brine layer and, thus, this depends primarily on the permeability of the medium. Downward migration is observed when the brine is static, at a permeability of $K=10^{-14}$ $\mathrm{m}^{2}$. On the other hand, upward migration occurs when the fluid in the brine layer convects at a permeability of $K=10^{-13} \mathrm{~m}^{2}$. The porosity of the medium does not influence these results.

Fig. 8 shows the (dimensionless) average chemical content of the system for all simulations as a function of time. The numbers in the figures show the excess salinity of the brine layer compared to seawater. Note the different time scales on the horizontal axes. As mentioned before, the permeabilities considered in this study are too low to be representative for those of sheeted dikes and pillow lavas present in the upper part of the domain. Therefore, the depletion times depicted in Fig. 8 give an indication of the relative importance of the various parameters rather than being truly indicative for natural ridge-crest systems. The depletion time of the brine layer scales directly with porosity and the excess salinity of the brine layer, while it is inversely related to permeability. The rate of brine depletion is defined by the slope of the curves. At low permeability, the depletion rate is lower and more uniform than the simulations at high permeability. In high-permeability media, the brine is quickly flushed out of the domain after a period with almost no salinity flux.

\section{Discussion and conclusions}

We have presented numerical simulations of the 

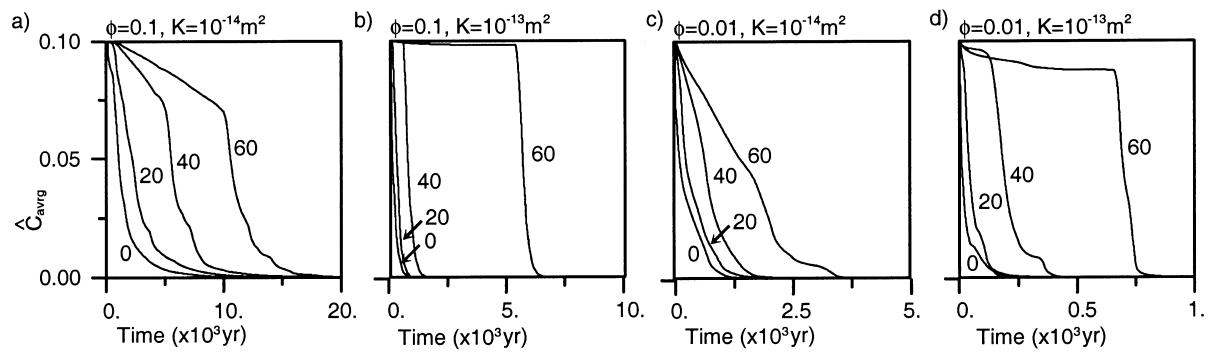

Fig. 8. The temporal evolution of the average chemical content of the system (dimensionless: initial value equals 0.1 ), plotted for various values of permeability and porosity: (a) $K=10^{-14} \mathrm{~m}^{2}$ and $\phi=0.1$, (b) $K=10^{-13} \mathrm{~m}^{2}$ and $\phi=0.1$, (c) $K=10^{-14} \mathrm{~m}^{2}$ and $\phi=0.01$, and (d) $K=10^{-13} \mathrm{~m}^{2}$ and $\phi=0.01$. The numbers denote the excess salinity of the brine layer as compared to seawater (the simulation with $\Delta C_{\mathrm{e}}=0 \mathrm{wt} \%$ resembles a passive tracer experiment).

evolution of a brine-saturated layer that has returned to the subcritical single-phase regime. In the simulations, the initial chemical content of the brine layer and the porosity and permeability of the basaltic crust were varied systematically. We have focused on the fluid-dynamical mechanisms associated with the depletion of the brine layer, the thermal and chemical signatures of the vent fluids and, finally, the time scales involved with the depletion.

For realistic geological parameters, the density contrast across the thermochemical interface between the brine layer and the overlying liquid is not large enough to prevent the convective currents from entraining fluid advectively across the interface between the two layers. This contradicts the conventional idea of a steady, layered doublediffusive system, in which heat and solute transport across the interface is purely diffusive/dispersive $[5,14,37,38]$. From his laboratory experiments, Griffiths [14] concluded that purely diffusive/dispersive interfaces can be maintained between two convective layers of different salinity. Our simulations differ from Griffiths' experiments, in the sense that both the porosity and the initial density difference between the two layers are lower. Schoofs et al. [18] argue that the stability of the interface is related directly to these quantities thus explain this scenery.

For our experiments, we observed two fluid-dynamical mechanisms by which the brine layer is depleted: convective breakdown and migration of the interface. The mechanism depends critically on the salinity of the brine layer and the convective forces within the adjacent layers.
The density interface was not very stable in situations where the fluid in the brine layer convects, leading to a quick disappearance of the interface. The type of mechanism which leads to the removal of the interface depends primarily on the brine salinity. The interface starts to deflect in systems where the salinity difference across the interface does not exceed $20 \mathrm{wt} \%$. The deflected interface breaks down when the bottom of the hydrothermal system is reached. For larger chemical contrasts between the convective brine and the overlying fluids, the brine layer is depleted by an upward migration of the interface.

For a static brine layer, the brine is entrained slowly by the fluid currents above the interface. As a result, the almost planar chemical interface migrates downward. The salinity of the vent fluids is approximately two times that of seawater, and this remains almost constant during the whole stage of depletion of the brine layer. During this period, the vent temperatures are slightly reduced. These observations are in reasonable agreement with measurements of the chronic ' $A$ ' and ' $F$ ' vents at $9^{\circ} 16.8^{\prime} \mathrm{N}$, East Pacific Rise [3].

The depletion times observed in our simulations are larger than those obtained from simple physical models of diffusion and dispersion across the interface [10]. We attribute this difference to the lower permeabilities used in our models. Under hydrological parameters similar to those of Lowell and Germanovich [10], the fluid in the brine layer will certainly convect. From our experiments, this implies that the brine layer will not be stable and the density interface will vanish either by convective breakdown or upward migra- 
tion, on a time scale much shorter than the one predicted for depletion across a purely diffusive/ dispersive interface [10].

From the experiments, we conclude that chronic venting of high-chlorinity fluids is indicative of the depletion of a static rather than convective brine layer. If two-phase separation continues to occur, a steady state brine layer may be preserved. This would be an alternative for the model of Bischoff and Rosenbauer [5].

We have to be careful with this interpretation, though, since our model only considers the subcritical liquid phase: the chronic high-salinity vents may also be a direct surface signal of ongoing phase separation. Furthermore, in the simulations in which the interface is not very stable, the depletion of the brine will certainly be influenced by the transition from super- to subcritical conditions. With this in mind, strong temporal variations in the vent salinity measured at a single site are merely due to the intrinsically chaotic behavior of the system [32], rather than by a (subcritical) depletion of an already developed brine layer. Finally, the results described here may also be relevant in back-arc spreading regions and in continental geothermal systems where supercritical phase separation is observed [39].

\section{Acknowledgements}

Ron Trompert is acknowledged for his efforts during the development of the numerical method. We thank William Wilcock and an anonymous reviewer for the constructive comments and Frank Spera, Jeroen van Hunen and Gualbert Oude Essink for the valuable discussions. The investigations were supported by the Research Council for Earth and Lifesciences (ALW/NWO), within the project 750.195.08. [EB]

\section{References}

[1] R.P. Lowell, P.A. Rona, R.P. Von Herzen, Seafloor hydrothermal systems, J. Geophys. Res. 100 (1995) 327-352.

[2] A. Schultz, J.R. Delaney, R.E. McDuff, On the partition- ing of heat flux between diffuse and point source seafloor venting, J. Geophys. Res. 97 (1992) 12299-12314.

[3] K.L. Von Damm, Controls on the chemistry and temporal variability of seafloor hydrothermal fluids, in: S.E. Humphris, R.A. Zierenberg, L.S. Mullineaux, R.E. Thomsen (Eds.), Seafloor Hydrothermal Systems: Physical, Chemical, Biological and Geological Interactions, Geophys. Monogr. Ser., 91, 222-247, AGU, Washington, DC, 1995.

[4] S.D. Scott, Submarine hydrothermal systems and deposits, in: H.L. Barnes (Ed.), Geochemistry of Hydrothermal Ore Deposits, 1997, pp. 797-875.

[5] J.L. Bischoff, R.J. Rosenbauer, Salinity variations in submarine hydrothermal systems by layered double-diffusive convection, J. Geol. 97 (1989) 613-623.

[6] C. Palliser, R. McKibbin, A model for deep geothermal brines, I: T-p-X state-space description, Transp. Porous Media 33 (1998) 65-80.

[7] C. Palliser, R. McKibbin, A model for deep geothermal brines, II: Thermodynamic properties - density, Transp. Porous Media 33 (1998) 129-154.

[8] C. Palliser, R. McKibbin, A model for deep geothermal brines, III: Thermodynamic properties - enthalpy and viscosity, Transp. Porous Media 33 (1998) 155-171.

[9] P. Nehlig, Salinity of oceanic hydrothermal fluids: a fluid inclusion study, Earth Planet. Sci. Lett. 102 (1991) 310 325.

[10] R.P. Lowell, L.N. Germanovich, Evolution of a brinesaturated layer at the base of a ridge-crest hydrothermal system, J. Geophys. Res. 102 (1997) 10245-10255.

[11] W.S.D. Wilcock, Cellular convection models of mid-ocean ridge hydrothermal circulation and the temperatures of black smoker fluids, J. Geophys. Res. 103 (1998) 2585 2596.

[12] D.A. Butterfield, G.J. Massoth, Geochemistry of north Cleft segment vent fluids: Temporal changes in chlorinity and their possible relation to recent volcanism, J. Geophys. Res. 99 (1994) 4951-4968.

[13] K.L. Von Damm, L.G. Buttermore, S.E. Oosting, A.M. Bray, D.J. Fornari, M.D. Lilley, W.C. Shanks III, Direct observation of the evolution of a seafloor 'black smoker' from vapor to brine, Earth Planet. Sci. Lett. 149 (1997) 101-111.

[14] R.W. Griffiths, Layered double-diffusive convection in porous media, J. Fluid Mech. 102 (1981) 221-248.

[15] W.E. Seyfried Jr., Experimental and theoretical constraints on hydrothermal alteration processes at midocean ridges, Annu. Rev. Earth Planet. Sci. 15 (1987) 317-335.

[16] W.E. Seyfried Jr., K. Ding, M.E. Berndt, Phase equilibria constraints on the chemistry of hot spring fluids at midocean ridges, Geochim. Cosmochim. Acta 55 (1991) 35593580 .

[17] S. Schoofs, R.A. Trompert, U. Hansen, The formation and evolution of layered structures in porous media, J. Geophys. Res. 103 (1998) 20843-20858.

[18] S. Schoofs, R.A. Trompert, U. Hansen, The formation 
and evolution of layered structures in porous media: Effects of porosity and mechanical dispersion, Phys. Earth Planet. Int. 118 (2000) 205-225.

[19] N.D. Rosenberg, F.J. Spera, R.M. Haymon, The relationship between flow and permeability field in seafloor hydrothermal systems, Earth Planet. Sci. Lett. 116 (1993) 135-153.

[20] A.R. Sohn, S.C. Webb, J.A. Hildebrand, B.D. Cornuelle, Three-dimensional tomographic velocity structure of the upper crust, CoAxial segment, Juan de Fuca Ridge: Implications for on-axis evolution and hydrothermal circulation, J. Geophys. Res. 102 (1997) 17679-17695.

[21] C.J. Richardson, J.R. Cann, H.G. Richards, J.G. Cowan, Metal-depleted root zones of the Troodos ore-forming hydrothermal systems, Earth Planet. Sci. Lett. 84 (1987) 243-253.

[22] H.G. Richards, J.R. Cann, J. Jensenius, Mineralogical zonation of metasomatism of alteration pipes in the Cyprus sulphide deposits, Econ. Geol. 84 (1989) 91-115.

[23] L.H. Kellogg, B.H. Hager, R.D. van der Hilst, Compositional stratification in the deep mantle, Science 283 (1999) 1881-1884.

[24] D.A. Nield, A. Bejan, Convection in Porous Media, Springer, New York, 1992.

[25] P. Nehlig, Fracture and permeability analysis in magmahydrothermal transition zones in the Samail ophiolite (Oman), J. Geophys. Res. 99 (1994) 589-601.

[26] A.T. Fisher, Permeability within the basaltic oceanic crust, Rev. Geophys. 36 (1998) 143-192.

[27] R.W. Potter II, D.L. Brown, The volumetric properties of aqueous sodium chloride solutions from $0^{\circ}$ to $500^{\circ} \mathrm{C}$ at pressures up to 2000 bars based on a regression of available data in literature, US Geol. Surv. Bull. 1421-C (1977) $36 \mathrm{pp}$.

[28] A.W. Herbert, C.P. Jackson, D.A. Lever, Coupled groundwater flow and solute transport with fluid density strongly dependent on concentration, Water Resour. Res. 24 (1988) 1781-1795.
[29] E. Holzbecher, Modeling Density-Driven Flow in Porous Media, Springer, Berlin, 1998, 286 pp.

[30] D. Norton, J. Knight, Transport phenomena in hydrothermal systems: cooling plutons, Am. J. Sci. 277 (1977) 937-981.

[31] H.C. Helgeson, D.H. Kirkham, Theoretical prediction of the thermodynamic behaviour of aqueous electrolytes at high pressures and temperatures, I, Am. J. Sci. 274 (1974) 1089-1198.

[32] S. Schoofs, F.J. Spera, U. Hansen, Chaotic thermohaline convection in low-porosity hydrothermal systems, Earth Planet. Sci. Lett. 174 (1999) 213-229.

[33] S. Schoofs, Thermochemical convection in porous media: An application to hydrothermal systems and magmatic intrusions, Ph.D. Dissertation, Utrecht University, The Netherlands, 1999.

[34] W.S.D. Wilcock, A. McNabb, Estimates of crustal permeability on the Endeavour segment of the Juan de Fuca mid-ocean ridge, Earth Planet. Sci. Lett. 128 (1995) 83-91.

[35] P. Nehlig, T. Juteau, V. Bendel, J. Cotten, The root zones of oceanic hydrothermal systems: constraints from the Samail ophiolite (Oman), J. Geophys. Res. 99 (1994) 4703-4713.

[36] J. Bear, Modeling flow and contaminant transport in fractured rocks, in: J. Bear, C.-F. Tsang, G. de Marsily (Eds.), Flow and Contaminant Transport in Fractured Rocks, Academic Press, San Diego, 1993, pp. 1-37.

[37] L.W. Gelhar, C. Welty, K.R. Rehfeldt, A critical review of data on field-scale dispersion in aquifers, Water Resour. Res. 28 (1992) 1955-1974.

[38] R.O. Fournier, Double-diffusive convection in geothermal systems: The Salton Sea, California, geothermal system as a likely candidate, Geothermics 19 (1990) 481-496.

[39] P.A. Rona, S.D. Scott, A special issue on seafloor hydrothermal mineralization: new perspectives, Econ. Geol. 88 (1993) 1935-1976. 